\title{
SYMMETRIES AND THE INVERSE PROBLEM OF LAGRANGIAN DYNAMICS FOR LINEAR SYSTEMS
}

\author{
W. SARLET ${ }^{\prime}$
}

(Received 20 July 1982)

\begin{abstract}
We discuss general, tume-dependent, linear systems of second-order ordinary differential equations. A study is made of the similarities and discrepancies between the inverse problem of Lagrangian mechanics on the one hand, and the search for linear dynamical symmetries on the other hand.
\end{abstract}

\section{Introduction}

We intend to discuss certain aspects of the search for dynamical symmetries of linear systems of second-order ordinary differential equations, particularly in relation to possible connections and analogies with the so-called inverse problem of Lagrangian mechanics for such systems.

The motivation for this paper is founded on a previous one, [8], in which we were able to disentangle a connection between the above mentioned inverse problem and the construction of quadratic first integrals for linear systems. Both of these problems can be viewed as a search for a time-dependent multiplier matrix and the observations made in [8] may roughly be summarized as follows. First of all, we can identify in a rather natural way some additional restrictions on the form of quadratic first integrals which automatically bring us to an interesting special case where a sufficiency criterion for existence of a Lagrangian is satisfied. Secondly, we can reformulate the general question of constructing quadratic integrals in such a way that the equations exhibit their deviation from the previous case. The special feature of this reformulated problem is that all coefficients appearing in the matrix differential equations now are expressed in

\footnotetext{
'Instituut voor Theoretische Mechanica, Rijksuniversiteit Gent, Krijgslaan 281 S-9, B-9000 Gent, Belgium.

(c) Copyright Australian Mathematical Society 1983, Serial-fee code 0334-2700/83.
} 
terms of the quantities which play a dominant role in the inverse problem of Lagrangian mechanics. The reformulated equations therefore are believed to be of particular interest in cases where a Lagrangian exists. More details about these observations will be recalled below.

It is well known that in the case of Lagrangian systems there is a duality between symmetries and first integrals which is provided by Noether's theorem. It is therefore not unreasonable to think that similar features should be observable with respect to the search for linear symmetries of the differential equations. Establishing these features is the object of the present paper. Since we do not in general require that the system be derivable from a variational principle, it is clear that a separate investigation of the symmetry aspect of the picture is needed, that is, the present study cannot amount to a mere transfer of the results in [8] with the aid of Noether's theorem.

In the next section, we recollect some relevant results from previous papers on the inverse problem for linear systems and we explicitly mention those equations from [8], which show the relation with the construction of quadratic first integrals. Section 3 contains the derivation of the similar occurrences in the study of linear symmetries. In Section 4 we are able to establish some deeper analogies (as well as discrepancies) by discussing how the "commutativity cases", introduced in the context of the inverse problem, also play a distinctive role in the context of linear point symmetries. We finally treat an example, for which we chose the time-dependent $n$-dimensional oscillator in order to have a further way of relating the present discussions to [8].

\section{The inverse problem for linear systems}

We consider linear systems of second-order equations, written in the form

$$
\ddot{q}+2 A(t) \dot{q}+B(t) q=0,
$$

where $A$ and $B$ are $n \times n$ matrices.

The inverse problem of Lagrangian mechanics for systems of type (1) has been thoroughly studied in [4], [10] and [7]. Concerning the inverse problem for general second-order systems, we can refer the reader to the recent contributions [2] and [6]. We here recall some results of [10] concerning the search for quadratic Lagrangians for (1).

Multiplying (1) by a non-singular, symmetric matrix $V(t)$ will produce a set of Euler-Lagrange equations if and only if

$$
\dot{V}=A^{T} V+V A,
$$




$$
V \Phi^{(0)}=\left(V \Phi^{(0)}\right)^{T},
$$

where $\Phi^{(0)}$ is a known matrix, defined by

$$
\Phi^{(0)}=B-A^{2}-\dot{A} .
$$

For $V$ to be symmetric, it is thereby sufficient that we impose the symmetry condition on its initial value $V\left(t_{0}\right)=S$. Furthermore, the algebraic condition (3) can be replaced by an infinite set of algebraic conditions on $S$, namely

$$
S \Phi^{(k)}\left(t_{0}\right)=\left(S \Phi^{(k)}\left(t_{0}\right)\right)^{T}, \quad k=0,1, \ldots, \infty,
$$

where the matrices $\Phi^{(k)}(t)$ are recursively defined by

$$
\Phi^{(k+1)}(t)=\dot{\Phi}^{(k)}(t)+\left(A \Phi^{(k)}-\Phi^{(k)} A\right) .
$$

We refer to [10] for a discussion of the relevance of conditions (5). Finally, it is worth mentioning that the solution of the differential equation (2) with initial value $S$ is of the form

$$
V=\left(\Omega^{-1}\right)^{T} S \Omega^{-1},
$$

where $\Omega(t)$ satisfies the equation

$$
\dot{\Omega}+A \Omega=0, \quad \Omega\left(t_{0}\right)=I \quad \text { (unit matrix). }
$$

The algebraic condition (3) accordingly transforms to

$$
S \overline{\Phi^{(0)}}=\left(S \overline{\Phi^{(0)}}\right)^{T}
$$

where $\bar{X}$ for a general matrix $X$ is defined as

$$
\bar{X}=\Omega^{-1} X \Omega \text {. }
$$

Underlying this reformulation of the conditions is the fact that the linear transformation

$$
q=\Omega(t) y
$$

takes the given system (1) into its canonical form

$$
\ddot{y}+\overline{\Phi^{(0)}} y=0,
$$

for which a multiplier necessarily has to be constant (see [10]).

In many situations of interest in engineering mechanics the system (1) is autonomous and the matrices $A$ and $B$ moreover commute. This commutativity property is sufficient for the existence of a Lagrangian [3]. For time-dependent systems, it can be generalized to the condition

$$
\Phi^{(1)}(t)=0
$$

which again is sufficient for existence of a solution $V$ of (2) and (3) (see [10]). Also of interest are the cases where

$$
\Phi^{(k)}(t)=0,
$$


for some $k>1$, because the infinite set of requirements (5) on $S$ then is reduced to a finite set. In analogy with (13), we referred to (14) as to a $k$ th-order commutativity condition. Actually, there is a slightly more general situation in which (5) reduces to a finite set of conditions, namely when we have an identity of the form

$$
\Phi^{(k+1)}(t)=\sum_{l=0}^{k} \lambda_{l}(t) \Phi^{(l)}(t)+\mu(t) I
$$

as has been shown in the context of general second-order systems in [6].

Let us now briefly talk about the aforementioned connections with the search for quadratic first integrals of (1). A general quadratic first integral can be written in the form

$$
F=\frac{1}{2}\left(\dot{q}^{T} V \dot{q}+q^{T} D \dot{q}+q^{T} Z q\right),
$$

where $V$ and $Z$ are symmetric matrices. The matrix $V(t)$ thereby need not satisfy the requirements (2) and (3) of the inverse-problem-multiplier. However, if we impose on $F$ the supplementary restrictions that $V$ satisfy the differential equation (2) (and therefore is a candidate for being a multiplier) and that $D(t)$ be of the form

$$
D=2 A^{T} V,
$$

then the requirement that $F$ be a first integral necessarily implies $\Phi^{(1)}(t)=0$, so that eventually $V$ will be a multiplier for (1) (see [8]). Secondly, if we keep the general situation, we can always write

$$
\dot{V}=A^{T} V+V A+W,
$$

which defines a symmetric matrix $W(t)$. As a result one can show that $D$ in general must have the form

$$
D=2 A^{T} V-W+R,
$$

where $R(t)$ is skew-symmetric. The matrices $W$ and $R$ clearly characterize the deviation from the preceding special case. The interesting thing about them is that in terms of these matrices, the conditions for $F$ to be a first integral become equations whose coefficients solely involve the $\Phi^{(0)}$ and $\Phi^{(1)}$ which dominate the inverse problem. If we make use of the reduction embodied in (7) and (8), these equations become

$$
\left.\begin{array}{r}
\hat{V}=\tilde{W}, \quad \dot{W}=\tilde{U} \\
\dot{U}=(\tilde{R}-2 \tilde{W}) \overline{\Phi^{(0)}}-\tilde{V} \overline{\Phi^{(1)}}+\text { transpose }, \\
\hat{R}=\left(\tilde{V} \overline{\Phi^{(0)}}\right)^{T}-\tilde{V} \overline{\Phi^{(0)}}
\end{array}\right\}
$$


where for a general matrix $Y(t)$ we set

$$
\tilde{Y}(t)=\Omega^{T} Y \Omega \text {. }
$$

For more details about the derivation of (20) and an interesting application, see [8]. In the next section we now turn to the investigation of linear dynamical symmetries for (1).

\section{Linear dynamical symmetries}

Let $\Gamma$ denote the second-order vector field governing the given system (1), i.e.

$$
\Gamma=\frac{\partial}{\partial t}+\dot{q}^{\prime} \frac{\partial}{\partial q^{i}}+\Lambda^{\prime} \frac{\partial}{\partial \dot{q}^{i}},
$$

with

$$
\Lambda^{\prime}(t, q, \dot{q})=-(2 A \dot{q}+B q)^{\prime} .
$$

A vector field $Y$ is called a symmetry of $\Gamma$ if

$$
[Y, \Gamma]=0 \text {, }
$$

where [,] denotes the Lie-bracket. When one is interested in infinitesimal transformations leaving the differential equations invariant, one can actually allow the right-hand side of (23) to be a multiple of $\Gamma$, which amounts to a change in the parametrization of the integral curves. For many purposes, however, one can consider symmetries as being equivalent if they differ by a multiple of $\Gamma$, so that there is no loss of generality in working with the simplest representative of each class, namely the genuine symmetry generator satisfying (23). For more details about this, for instance in the context of Noether's theorem, see [9] and [5]. Writing for $Y$ an expression like

$$
Y=\mu^{i} \frac{\partial}{\partial q^{i}}+\nu^{i} \frac{\partial}{\partial \dot{q}^{\prime}}
$$

the symmetry requirement (23) is equivalent to

$$
\nu^{\prime}=\Gamma\left(\mu^{\prime}\right), \quad \Gamma\left(\nu^{\prime}\right)=Y\left(\Lambda^{\prime}\right) .
$$

Because of the linearity of our actual system (1), we intend to look now only at linear symmetries. To be specific, we assume that the components $\mu^{\prime}$ form a vector of the form

$$
\mu=-(P(t) q+Q(t) \dot{q}) .
$$

The $\nu^{\prime}$, in view of (25a), then necessarily must be of the form

$$
\nu=(2 Q A-\dot{Q}-P) \dot{q}+(Q B-\dot{P}) q,
$$


while (25b) requires $P$ and $Q$ to satisfy the following equations:

$$
\begin{gathered}
\ddot{Q}=4\left(\dot{Q} A+A Q A-Q A^{2}\right)+2(Q \dot{A}-A \dot{Q}) \\
+Q B-B Q+2(P A-A P-\dot{P}), \\
\ddot{P}=2(\dot{Q} B+A Q B-Q A B)+Q \dot{B}-2 A \dot{P}+P B-B P .
\end{gathered}
$$

At this point, we may simplify the equations a bit by making transitions of the type (10) which are related to the reduction of (1) to its canonical form (12). After some straightforward calculations, it is seen that (28) and (29) are equivalent to the following equations:

$$
\begin{aligned}
& \ddot{\bar{Q}}=2(\dot{\bar{Q}} \bar{A}+\bar{Q} \overline{\dot{A}}-\dot{\bar{P}})+\bar{Q} \overline{\Phi^{(0)}}-\overline{\Phi^{(0)}} \bar{Q}, \\
& \ddot{\bar{P}}=-2 \dot{\bar{P}} \bar{A}+2 \dot{\bar{Q}} \bar{B}+\bar{Q} \dot{\bar{B}}+\bar{P} \overline{\Phi^{(0)}}-\overline{\Phi^{(0)}} \bar{P}
\end{aligned}
$$

where in passing we note that

$$
\overline{\dot{A}} \equiv \dot{\bar{A}}
$$

whereas, in general,

$$
\dot{\bar{X}}=\overline{\dot{X}}+[\bar{A}, \bar{X}]
$$

In looking for particular solutions of (30) and (31), it is quite natural to start with the choice $Q=I(\Leftrightarrow \bar{Q}=I)$, because this means that to within a rescaling of the time-variable we are restricting ourselves to a class of point-symmetries. With that choice, (28) or (30) reveal a simple particular solution for $P$, namely $P=A$. The second equation to be satisfied, i.e. (29) or (31), then necessarily implies (13), so that we are in a situation where a multiplier $V$ for the inverse problem exists. This is of course the complete analogue of the observation made around equation (17), concerning the construction of quadratic first integrals. We may summarize the statement as follows.

Proposition 1. Equation (1) will have a linear point symmetry $Y$, governed by the choice

$$
\mu=-A q-\dot{q}
$$

if and only if $\Phi^{(1)}=0$. We then have a Lagrangian for the system, with respect to which $Y$ is a Noether-symmetry and as such produces a quadratic first integral, described in [8] and [10].

The reader will have no trouble verifying the last part of this statement after going through the example at the end of the paper. 
Next, we turn back to the general equations (30), (31) and make the following substitution,

$$
\bar{P}=\bar{Q} \bar{A}+\bar{T},
$$

which defines the matrix $\bar{T}$. This substitution should be viewed as characterizing the deviation from the preceding special case, in analogy with the substitution (18), (19) concerning quadratic integrals. It is straightforward to compute that (30), (31), in view of (34) and making use of the properties (32), (33), reduce to

$$
\begin{gathered}
\ddot{\bar{Q}}=\bar{Q} \overline{\Phi^{(0)}}-\overline{\Phi^{(0)}} \bar{Q}-2 \dot{\bar{T}}, \\
\ddot{\bar{T}}+\overline{\Phi^{(0)}} \bar{T}-\bar{T} \overline{\Phi^{(0)}}=2 \dot{\bar{Q}} \overline{\Phi^{(0)}}+\bar{Q} \overline{\Phi^{(1)}} .
\end{gathered}
$$

Note the appearance of the quantities $\Phi^{(0)}$ and $\Phi^{(1)}$ in this reduced general system. The analogy with the study of quadratic integrals could even be made more transparent by replacing the two second-order equations (35), (36) by four first-order equations and comparing these with equations (20). Summarizing again, we come to the following

Proposition 2. In order to find a linear dynamical symmetry of (1), one must find a solution $(\bar{Q}, \bar{T})$ of equations (35), (36). With the help of such a solution, the symmetry is determined by

$$
\mu=-(Q A+T) q-Q \dot{q},
$$

where the barred matrices are related to the unbarred ones by the similarity transformation (10).

\section{Some further analogies with the inverse problem}

Let us return to the class of point symmetries, for which we have $Q=I$. The general equations (35), (36) then reduce to

$$
\begin{gathered}
\dot{\bar{T}}=0, \\
\bar{T} \overline{\Phi^{(0)}}-\overline{\Phi^{(0)}} \bar{T}+\overline{\Phi^{(1)}}=0 .
\end{gathered}
$$

Equation (37) implies that $\bar{T}$ is constant, say

$$
\bar{T}=T_{0} \text {. }
$$

Assuming the given matrices $A$ and $B$ to be real analytic in the neighborhood of $t_{0}$, (38) can be replaced by an infinite set of algebraic conditions $t=t_{0}$, coming from the coefficients of its Taylor expansion. Recalling the definition (6) of the 
matrices $\Phi^{(k)}(t)$ one readily finds that these conditions read (remember that $\left.\Omega\left(t_{0}\right)=I\right)$

$$
T_{0} \Phi^{(k)}\left(t_{0}\right)-\Phi^{(k)}\left(t_{0}\right) T_{0}+\Phi^{(k+1)}\left(t_{0}\right)=0, \quad \forall k .
$$

A further analogy now emerges with the necessary and sufficient conditions (5) arising in the study of the inverse problem. Assume for instance that one of the "higher order commutativity conditions" is satisfied, say

$$
\phi^{(k+1)}(t) \equiv 0 \text {. }
$$

Since this implies $\Phi^{(k+m)}(t)=0(m \geqslant 1)$, the system (40) reduces to a finite number of equations, the last of which reads

$$
T_{0} \Phi^{(k)}\left(t_{0}\right)-\Phi^{(k)}\left(t_{0}\right) T_{0}=0
$$

It is then, in principle, a very straightforward matter to find out whether the given system has linear point symmetries. Let us further look for an analogue of the more general situation (15). Assuming (15) to hold true for certain functions $\lambda_{l}(t)$ and $\mu(t)$, we can first of all easily check that a similar linear dependence will occur for all further $\Phi^{(k+m)}$. For example, we have

$$
\Phi^{(k+2)}(t)=\sum_{l=0}^{k} \lambda_{l} \Phi^{(l+1)}+\sum_{l=0}^{k} \dot{\lambda}_{l} \Phi^{(l)}+\dot{\mu} I .
$$

Suppose then that we have

$$
T_{0} \Phi^{(l)}\left(t_{0}\right)-\Phi^{(l)}\left(t_{0}\right) T_{0}+\Phi^{(l+1)}\left(t_{0}\right)=0
$$

for $l=0,1, \ldots, k$. Multiplying (44) by $\lambda_{l}\left(t_{0}\right)$ and summing for $l$ ranging from 0 to $k$, we obtain (using (15))

$$
T_{0} \Phi^{(k+1)}\left(t_{0}\right)-\Phi^{(k+1)}\left(t_{0}\right) T_{0}+\sum_{l=0}^{k} \lambda_{l}\left(t_{0}\right) \Phi^{(l+1)}\left(t_{0}\right)=0 .
$$

If we want this to imply (44) for $l=k+1$, we should have

$$
\Phi^{(k+l)}\left(t_{0}\right)=\sum_{l=0}^{k} \lambda_{l}\left(t_{0}\right) \Phi^{(l+l)}\left(t_{0}\right)
$$

Comparing (46) with (43) and keeping in mind that the same result should apply for all further $\Phi^{(k+m)}$, we necessarily must require $\lambda_{l}$ and $\mu$ to be constant. Summarizing, we have shown:

Proposition 3. Assume that the given matrices $A$ and $B$ satisfy the relation

$$
\Phi^{(k+1)}(t)=\sum_{l=0}^{k} \lambda_{l} \Phi^{(l)}(t)+\mu I
$$


where $\lambda$, and $\mu$ are constants. Then equation (1) will have a linear point symmetry (of type $Q=I$ ) iff a solution $T_{0}$ exists of he algebraic equations (44), for $l=0,1, \ldots, k$. Such a point symmetry is given by a vector field $Y$ of type (24), with $\mu$ determined by

$$
\mu=-\left(A+\Omega T_{0} \Omega^{-1}\right) q-\dot{q},
$$

$\Omega(t)$ being the solution of $(8)$.

The cases of higher-order commutativity are of course recovered in this statement for $\lambda_{1}=\mu=0$. If in addition we take $k=0$, we get back to the situation described in Proposition 1.

We have of course, despite the analogy, also discovered a discrepancy here between the study of the inverse problem and the study of linear point symmetries, since the $\lambda_{l}$ and $\mu$ here have to be constant. A much more fundamental discrepancy, however, is the following one. In looking for a multiplier matrix in the inverse problem, we generally have to deal with symmetry requirements of the product of two matrices. In the search for linear point symmetries we encounter commutativity conditions instead. This is for instance very striking when one compares equations (5) with (40) and (42). There is one particularly interesting exception in this respect, namely the case that (1) in its given form is already a set of Euler-Lagrange equations (i.e. has $I$ as a multiplier). Indeed, in that case $A$ is skew-symmetric and all $\Phi^{(k)}$ accordingly become symmetric, so that symmetry requirements of the type (5) reduce to commutativity requirements (see also [6]).

As a final remark for this section, in case Proposition 3 cannot be applied, note that there is still some interest in the infinite set of conditions (40) (similar to the interest of (5) in the inverse problem). Indeed, (40) produces an infinite set of linear algebraic equations for the finite number of elements of the unknown matrix $T_{0}$. So, it is in principle possible to derive from these equations some further consistency conditions for existence of a solution which would then be expressed in terms of known elements only and this would allow the verification of certain necessary conditions for existence of a linear point symmetry, prior to its actual construction.

\section{An example}

For the example, we go back to Proposition 2, i.e., to the general equations (35), (36) for the determination of a linear symmetry. Because of the appearance of the quantities $\Phi^{(0)}$ and $\Phi^{(1)}$ in these equations, it is conceivable that they are easier to tackle than the original equations (28), (29) in cases where a Lagrangian 
is known to exist. To illustrate their elegance in certain applications and to allow a further comparison with the results of [8], we choose the same simple example of an $n$-dimensional, time-dependent, isotropic oscillator. The equations of motion are

$$
\ddot{q}_{t}+\omega^{2}(t) q_{i}=0 .
$$

We have $\Phi^{(0)}=\omega^{2}(t) I, A=0, \Omega=I$, so that the general equations (35), (36) simplify to

$$
\begin{gathered}
\ddot{Q}=-2 \dot{T}, \\
\ddot{T}=2 \omega^{2} \dot{Q}+2 \omega \dot{\omega} Q .
\end{gathered}
$$

Elimination of $T$ between these equations leads to the third-order equation

$$
\ddot{Q}+4 \omega^{2} \dot{Q}+4 \omega \dot{\omega}=0 .
$$

In order to find particular solutions of (49), we try $Q(t)=\gamma(t) Q_{0}$, where $\gamma$ is a scalar function and $Q_{0}$ is constant. The function $\gamma$ then must satisfy a similar third-order equation, which by the substitution $\gamma=\rho^{2}$ can be integrated to

$$
\ddot{\rho}+\omega^{2} \rho=c \rho^{-3} .
$$

The first of equations (48) integrates to

$$
\dot{Q}=-2 T+2 T_{0}
$$

Hence, with

$$
Q=\rho^{2} Q \quad \text { and } T=T_{0}-\rho \dot{\rho} Q_{0},
$$

we get a set of solutions of (48), $Q_{0}$ and $T_{0}$ being arbitrary constant $n \times n$ matrices and $\rho$ satisfying the scalar equation (50). A class of linear dynamical symmetries therefore is determined by

$$
\mu=-\left(T_{0}-\rho \dot{\rho} Q_{0}\right) q-\rho^{2} Q_{0} \dot{q} .
$$

Because of the arbitrariness of $Q_{0}$ and $T_{0},(52)$ actually gives a $2 n^{2}$-parameter family of linear symmetries. It is known that such symmetries in general do not produce a corresponding first integral by simple algebraic means. In order to do so, they must be of Noether-type. So let us investigate under which supplementary restrictions on $T_{0}$ and $Q_{0}(52)$ will produce a Noether-symmetry with respect to the Lagrangian

$$
L=\frac{1}{2} \dot{q}^{T} \dot{q}-\frac{1}{2} \omega^{2} q^{T} q
$$

for the present problem. The criterion for the vector field $Y$ to be a Noether-symmetry with respect to $L$ is that the one-form $i_{Y} d \theta(L)$ be exact, where $\theta(L)$ is the so-called Cartan-form and $i_{Y}$ stands for the inner product of the vector field $Y$ with differential forms (see [9]). We have

$$
i_{Y} d \theta=\nu^{\prime}\left(d q^{\imath}-\dot{q}^{\prime} d t\right)-\mu^{\prime}\left(d \dot{q}^{\prime}-\Lambda^{i} d t\right),
$$


where $\Lambda^{i}$ for the present case is equal to $-\omega^{2} q$, and the components $\nu^{i}$, in agreement with (27), are given by

$$
\nu=-\left(T_{0}+\rho \dot{\rho} Q_{0}\right) \dot{q}+\left(\dot{\rho}^{2}+\rho^{-2}\right) Q_{0} .
$$

The one-form (53) will equal $d F$ for some function $F$ if and only if

$$
\nu=\frac{\partial F}{\partial \dot{q}} \quad \text { and } \quad-\mu=\frac{\partial F}{\partial \dot{q}} .
$$

Using the explicit forms (52) and (54), the integrability conditions for the validity of (55) are obtained as

$$
Q_{0}=Q_{0}^{T}, \quad-\left(T_{0}+\rho \dot{\rho} Q_{0}\right)=\left(T_{0}-\rho \dot{\rho} Q_{0}\right)^{T} .
$$

They are equivalent to

$$
Q_{0}=Q_{0}^{T} \quad \text { and } \quad T_{0}=-T_{0}^{T} .
$$

Finally, the corresponding class of first integrals is given by

$$
F=\frac{1}{2}\left(\dot{\rho}^{2}+\rho^{-2}\right) q^{T} Q_{0} q-q^{T}\left(T_{0}+\rho \dot{\rho} Q_{0}\right) \dot{q}+\frac{1}{2} \rho^{2} \dot{q}^{T} Q_{0} \dot{q},
$$

$Q_{0}$ being arbitrary symmetric and $T_{0}$ arbitrary skew-symmetric. As already discussed in [8], (57) contains in one single formula all quadratic first integrals discussed by Günther and Leach [1].

\section{Some final comments}

All results of this paper have been extensively discussed throughout the text. We therefore limit ourselves here to some additional suggestions for further work. The close similarities between the inverse problem of Lagrangian mechanics and the search for symmetries, discussed in Section 4, seem to open the way for a classification type study. In particular, we are thinking of the cases where a commutativity condition like (41) is satisfied. Starting with two degrees of freedom, one could for instance list all second-order systems for which $\Phi^{(1)}=0$, $\Phi^{(2)}=0$, etc. For each of these cases, one could then separately investigate the existence of a multiplier on the one hand (a finite set of conditions of type (5)) and the existence of linear point symmetries on the other hand (a finite set of conditions of type (40) $)$ in the hope of coming to a better understanding of the interconnections and discrepancies between both problems and the significance of the quantities $\Phi^{(k)}(t)$.

As a final comment, it is worthwhile indicating that the restriction to linear systems in this paper should not be regarded as oversimplification. First of all, there still is a lot of interest in linear systems on their own in various branches of 
applied mathematics. Secondly, it has been experienced by us that the study of the inverse problem of Lagrangian mechanics for linear systems [10], provides valuable insight for tackling the case of general second-order equations [6]. It is for instance well known that a general second-order system is of Lagrangian type if and only if the same is true for its linear variational equations.

\section{Acknowledgements}

This work was completed while the author was a Visiting Research Fellow at the Department of Applied Mathematics of La Trobe University during the summer/winter of 1982 . He gratefully acknowledges the support of the Potter foundation and La Trobe University. He is further indebted to Dr. P. G. L. Leach and Dr. F. Cantrijn for stimulating discussion and to Professor R. Mertens for his continuing support.

\section{References}

[1] N. J. Günther and P. G. L. Leach, "Generalized invariants for the time-dependent harmonic oscillator", J. Math. Phys. 18 (1977), 572-576.

[2] M. Henneaux, "Equations of motion, commutation relations and ambiguities in the Lagrangian formulism", Ann. Physics 140 (1982), 45-64.

[3] H. G. Kwatny, L. Y. Bahar and F. M. Massimo, "Linear non-conservative systems with asymmetric parameters derivable from a Lagrangian", Hadronic J. 2 (1979), 1159-1177.

[4] L. A. Novak and M. M. Milić, "On the existence of variational formulation for gencral time-varying systems", Proc 1980 IEEE Internat. Symp. on Circuts and Systems (Houston, Texas) (1980), 830-832.

[5] W. Sarlet, "Symmetries, first integrals and the inverse problem of Lagrangian mechanics", $J$. Phys. A 14 (1981), 2227-2238.

[6] W. Sarlet, "The Helmholtz conditions revisited. A new approach to the inverse problem of Lagrangian dynamics", J. Phys. A 15 (1982), 1503-1517.

[7] W. Sarlet. "Note on linear systems derivable from a variational principle", Phys. Lett.. (to appear).

[8] W. Sarlet and L. Y. Bahar, "Quadratic integrals for linear non-conservative systems and their connection with the inverse problem of Lagrangian dynamics", Internat. $J$ Non-linear Mech. 16 (1981). 271-281.

[9] W. Sarlet and F. Cantrijn, "Generalizations of Noether's theorem in classical mechanics", SIAM Rev. 23 (1981), 467-494.

[10] W. Sarlet, E. Engels and L. Y. Bahar, "Time-dependent linear systems derivable from a variational principle", Internat. J. Engrg. Sci. 20 (1982), 55-66. 